\title{
Chemical patterns of colony membership and mother- offspring similarity in Antarctic fur seals are reproducible
}

\author{
Jonas Tebbe $^{1}$, Emily Humble ${ }^{1,2,3}$, Martin Adam Stoffel ${ }^{1,4}$, Lisa Johanna Tewes ${ }^{5}$, Caroline Müller ${ }^{5}$, Jaume Forcada ${ }^{3}$ \\ , Barbara Caspers ${ }^{6}$, Joseph Ivan Hoffman ${ }^{\text {Corresp. } 1,3}$ \\ ${ }^{1}$ Department of Animal Behaviour, Bielefeld University, Bielefeld, Germany \\ 2 Royal (Dick) School of Veterinary Studies and the Roslin Institute, University of Edinburgh, Edinburgh, United Kingdom \\ 3 British Antarctic Survey, Cambridge, United Kingdom \\ 4 Institute of Evolutionary Biology, University of Edinburgh, Edinburgh, United Kingdom \\ 5 Department of Chemical Ecology, Bielefeld University, Bielefeld, Germany \\ 6 Department of Behavioural Ecology, Bielefeld University, Bielefeld, Germany \\ Corresponding Author: Joseph Ivan Hoffman \\ Email address: joseph.hoffman@uni-bielefeld.de
}

Replication studies are essential for evaluating the validity of previous research findings. However, it has proven challenging to reproduce the results of ecological and evolutionary studies, partly because of the complexity and lability of many of the phenomena being investigated, but also due to small sample sizes, low statistical power and publication bias. Additionally, replication is often considered too difficult in field settings where many factors are beyond the investigator's control and where spatial and temporal dependencies may be strong. We investigated the feasibility of reproducing original research findings in the field of chemical ecology by performing an exact replication of a previous study of Antarctic fur seals (Arctocephalus gazella). In the original study, skin swabs from 41 mother-offspring pairs from two adjacent breeding colonies on Bird Island, South Georgia, were analysed using gas chromatography-mass spectrometry. Seals from the two colonies differed significantly in their chemical fingerprints, suggesting that colony membership may be chemically encoded, and mothers were also chemically similar to their pups, hinting at the possible involvement of phenotype matching in mother-offspring recognition. In the current study, we generated and analysed chemical data from a nonoverlapping sample of 50 mother-offspring pairs from the same two colonies five years later. The original results were corroborated in both hypothesis testing and estimation contexts, with $p$-values remaining highly significant and effect sizes, standardized between studies by bootstrapping the chemical data over individuals, being of comparable magnitude. However, exact replication studies are only capable of showing whether a given effect can be replicated in a specific setting. We therefore investigated whether chemical signatures are colony-specific in general by expanding the geographic coverage 
of our study to include pups from a total of six colonies around Bird Island. We detected significant chemical differences in all but a handful of pairwise comparisons between colonies. This finding adds weight to our original conclusion that colony membership is chemically encoded, and suggests that chemical patterns of colony membership not only persist over time but can also be generalized over space. Our study systematically confirms and extends our previous findings, while also implying more broadly that spatial and temporal heterogeneity need not necessarily negate the reproduction and generalization of ecological research findings. 
1 Chemical patterns of colony membership and mother-

2 offspring similarity in Antarctic fur seals are

\section{3 reproducible}

4

5 Jonas Tebbe ${ }^{1}$, Emily Humble ${ }^{1,2,3}$, Martin Adam Stoffel ${ }^{1,4}$, Lisa Johanna Tewes ${ }^{5}$, Caroline

6 Müller $^{5 *}$, Jaume Forcada ${ }^{3 *}$, Barbara Caspers ${ }^{6 *} \&$ Joseph Ivan Hoffman ${ }^{1,3 *}$

7

81 Department of Animal Behaviour, Bielefeld University, 33501 Bielefeld, Germany

92 Royal (Dick) School of Veterinary Studies and the Roslin Institute, University of Edinburgh,

10 Edinburgh, EH25 9RG, UK

113 British Antarctic Survey, High Cross, Madingley Road, Cambridge CB3 OET, UK

124 Institute of Evolutionary Biology, University of Edinburgh, Edinburgh, EH9 3FL, UK

135 Department of Chemical Ecology, Bielefeld University, 33501 Bielefeld, Germany

146 Department of Behavioural Ecology, Bielefeld University 33501 Bielefeld, Germany

$15 *$ Jointly supervised this research

16

17 Corresponding Author:

18 Joseph Ivan Hoffman ${ }^{1}$

19 Department of Animal Behaviour, Bielefeld University, 33501 Bielefeld, Germany

20 Email address: joseph.hoffman@uni-bielefeld.de 


\section{Abstract}

23

24

25

26

27

28

29

30

31

32

33

34

35

36

37

38

39

40

41

42

43

44

45

46

47

48

49

50

51

Replication studies are essential for evaluating the validity of previous research findings. However, it has proven challenging to reproduce the results of ecological and evolutionary studies, partly because of the complexity and lability of many of the phenomena being investigated, but also due to small sample sizes, low statistical power and publication bias. Additionally, replication is often considered too difficult in field settings where many factors are beyond the investigator's control and where spatial and temporal dependencies may be strong. We investigated the feasibility of reproducing original research findings in the field of chemical ecology by performing an exact replication of a previous study of Antarctic fur seals (Arctocephalus gazella). In the original study, skin swabs from 41 mother-offspring pairs from two adjacent breeding colonies on Bird Island, South Georgia, were analysed using gas chromatography-mass spectrometry. Seals from the two colonies differed significantly in their chemical fingerprints, suggesting that colony membership may be chemically encoded, and mothers were also chemically similar to their pups, hinting at the possible involvement of phenotype matching in motheroffspring recognition. In the current study, we generated and analysed chemical data from a non-overlapping sample of 50 mother-offspring pairs from the same two colonies five years later. The original results were corroborated in both hypothesis testing and estimation contexts, with $p$-values remaining highly significant and effect sizes, standardized between studies by bootstrapping the chemical data over individuals, being of comparable magnitude. However, exact replication studies are only capable of showing whether a given effect can be replicated in a specific setting. We therefore investigated whether chemical signatures are colony-specific in general by expanding the geographic coverage of our study to include pups from a total of six colonies around Bird Island. We detected significant chemical differences in all but a handful of pairwise comparisons between colonies. This finding adds weight to our original conclusion that colony membership is chemically encoded, and suggests that chemical patterns of colony membership not only persist over time but can also be generalized over space. Our study systematically confirms and extends our previous findings, while also implying more 
52 broadly that spatial and temporal heterogeneity need not necessarily negate the 53 reproduction and generalization of ecological research findings.

54 


\section{Introduction}

56 Replication studies are fundamental to the scientific process as they are essential for evaluating 57 the correctness of scientific claims and the conclusions of other scientists (Schmidt, 2009). Indeed, 58 Fisher (1974) recommended that a null hypothesis should always be rejected more than once 59 because "no isolated experiment, however significant in itself, can suffice for the experimental 60 demonstration of any natural phenomenon". Nevertheless, replication studies are still "troublingly 61 rare”, particularly in fields such as ecology and evolutionary biology (Nakagawa \& Parker, 2015). 62 Palmer (2000) argued that we ignore reproducibility at our peril because this perpetuates a 63 "contract of error" that undermines our understanding of important ecological and evolutionary 64 phenomena.

65

66 There has also been debate and confusion over exactly what constitutes reproducible research 67 (Mendoza \& Garcia, 2017). Goodman and colleagues (2016) recognized three basic concepts, (i) 68 "methods reproducibility", which requires that the methodology of a given study be provided in 69 sufficient detail to allow it to be repeated; (ii) "results reproducibility", often known as "replication", which is the ability to corroborate previous results using the same experimental methods in a new study; and (iii) "inferential reproducibility", which relates to whether or not qualitatively similar conclusions are reached on the basis of either an independent replication of a study or a re-analysis of the original data. Furthermore, replication studies can be "exact", meaning

74 that they show a high degree of fidelity to the original experiment, "partial", which involves procedural or methodological changes, or "conceptual”, where the same questions are investigated but using different approaches (Kelly, 2006). The latter two categories include "quasi-replication" studies, which extend the scope of the original study beyond the specific system or species in question (Palmer, 2000). In general, the closer the replication attempt is to the original study, the more valuable are the results for assessing the validity of the original claims (Nakagawa \& Parker, 2015). However, quasi and conceptual replications are also important because they can shed light on the generality (also known as "transportability") of the effects under investigation (Goodman,

82 Fanelli \& Ioannidis, 2016; Dirnagl, 2019; Piper et al., 2019; although see Kelly, 2006). Put another 83 way, it is only possible to learn something about the broader significance of a certain effect by 84 probing to what extent it persists in settings that are different from, or which lie outside of the 
85

86

87

88

89

90

91

92

93

94

95

96

97

98

99

100

101

102

103

104

105

106

107

108

109

110

111

112

113

114

115

experimental framework of the original study. Quasi and conceptual replications therefore play an important role in increasing the "external validity" of results (Schmidt, 2009).

Another conceptual difficulty relates to the basis on which replication success is judged. Although there is no single standard for evaluating replication outcomes, most replication attempts are deemed successful if a null hypothesis that was rejected in the original study is again rejected (Rosenthal, 1991; Kelly, 2006). However, due to the dependence of $p$-values on sample sizes, success or failure in attaining significance may not always provide a good measure of replication success (Kelly, 2006). Consequently, several authors have advocated reporting effect sizes and associated measures of precision, as these allow replication outcomes to be gauged in a continuous manner rather than on the basis of binary significance outcomes (Kelly, 2006; Goodman, Fanelli \& Ioannidis, 2016; Piper et al., 2019).

In recent years, high-profile failures to reproduce a significant proportion of studies in the medical and social sciences (e.g. Begley \& Ellis, 2012; Open science collaboration, 2015, reviewed by Kelly, 2019) have led to a crisis of confidence (Baker, 2016). The generally poor success of replication studies has been attributed to a "publish or perish" culture that incentivizes dubious research practices such as selectively reporting significant results, $p$-value hacking and establishing hypotheses after the results of a study are known (Fidler et al., 2017; Fraser et al., 2018). All of these practices increase the risk of false positives and contribute towards publication bias (Jennions \& Møller, 2002), which undermines the robustness of the scientific literature. Further issues include poor study design, low statistical power, variability in reagents or the use of specialized techniques that are difficult to repeat, lack of scientific oversight, inadequate reporting of data, methods and results, and insufficient incentives for sharing data and code (Baker, 2016; Fidler et al., 2017; Piper et al., 2019).

11 Despite growing awareness of these issues not being specific to any particular scientific field, ecological and evolutionary studies are seldom replicated, with only around $0.02 \%$ of studies having been self-reported as exact replications (Kelly, 2019). One reason for this may be the general perception that research in these fields can be difficult to replicate, partly due to the complexity and lability of many of the phenomena under investigation, but also because in many 
116 field situations replication may be unfeasible or even unethical (Kelly, 2006; Nakagawa \& Parker, 117 2015; Fidler et al., 2017). Furthermore, numerous factors cannot be controlled for in natural 118 settings and environmental variation in particular may confound attempts to reproduce previous 119 results (Kelly, 2006). However, these are not valid reasons to neglect replication studies as it is 120 important to understand the extent to which research outcomes hinge upon these and other factors. 121

122 The field of chemical ecology provides an interesting case in point. Increasing numbers of studies 123 are using approaches like gas chromatography-mass spectrometry (GC-MS) to characterize the 124 chemical composition of biological samples such as skin swabs or urine. The resulting "chemical 125 fingerprints", otherwise commonly referred to as "chemical profiles", "scent profiles" or "odour 126 profiles" (Hurst \& Beynon, 2010), comprise multiple peaks that are separated according to their 127 retention times and which represent different substances. Studies of both captive and wild animal 128 populations have shown that these chemical fingerprints can convey information about species 129 identity (Caspers et al., 2009; Fratini et al., 2012; Krause et al., 2014), population membership 130 (Schneeberger et al., 2016; Wierucka et al., 2019), sex, age and reproductive state (Caspers et al., 131 2011; Kean, Müller \& Chadwick, 2011; Vogt et al., 2016), family membership (Sun \& Müller132 Schwarze, 1998; Müller \& Müller, 2016), individual identity (Kean, Chadwick \& Mueller, 2015; 133 Kohlwey et al., 2016), social status (Burgener et al., 2009) and genotype (Yamazaki et al., 1990; 134 Charpentier, Boulet \& Drea, 2008; Setchell et al., 2011). However, concerns have been raised 135 over the small sample sizes of many studies, which afford little statistical power and may 136 ultimately lead to effect sizes being overestimated (Wyatt, 2015). Furthermore, GC-MS data are 137 inherently noisy, making peak detection and alignment challenging (Ottensmann et al., 2018). The 138 failure to report peak detection and alignment methods in sufficient detail might therefore act as a 139 barrier to the successful replication of chemical studies. Finally, chemical fingerprints are complex 140 and multidimensional, being influenced by a multitude of factors (Hurst \& Beynon, 2010; Stoffel 141 et al., 2015) including both intrinsic (e.g. genes, hormones and metabolic status) and extrinsic (e.g. 142 environmental variation and diet) variables. Consequently, it remains unclear to what extent many 143 chemical patterns will be repeatable, particularly under natural and often highly heterogeneous 144 conditions. 
146 Pinnipeds provide interesting model systems for studying chemical communication as they possess 147 large repertoires of functional olfactory receptor genes (Kishida et al., 2007) and are sensitive to 148 even the faintest of smells (Kowalewsky et al., 2006). Many pinnipeds have a strong musky smell 149 (Hamilton, 1956), which has been attributed to facial glands that show hypertrophy during the 150 breeding season (Ling, 1974; Hardy et al., 1991), suggesting an important role of olfactory 151 communication during the peak reproductive period. Olfaction may be particularly crucial for 152 mother-offspring recognition because females of many pinniped species accept or reject pups after 153 naso-nasal inspection (Kovacs, 1995; Dobson \& Jouventin, 2003; Phillips, 2003). For example, a 154 study of Australian sea lions showed that mothers are capable of discriminating their own pups 155 from nonfilial conspecifics based on odour alone (Pitcher et al., 2011). This discovery motivated 156 our team to perform a study of Antarctic fur seals (Arctocephalus gazella), in which chemical 157 fingerprints were characterized from skin swabs taken from 41 mother-offspring pairs at two 158 breeding colonies-the special study beach (SSB) and freshwater beach (FWB)-at Bird Island, 159 South Georgia (Stoffel et al., 2015). Despite being separated by less than 200m, animals from 160 these two colonies exhibited highly significant chemical differences, while mothers showed greater chemical similarity to their pups than expected by chance.

162

163 Although further research is needed, these findings may have implications for the social 164 organization of Antarctic fur seals as well as for individual recognition. On the one hand, chemical 165 differences between animals from different colonies could potentially facilitate colony recognition and thereby help to explain the remarkable natal philopatry and site fidelity of this species (Hoffman et al. 2006; Hoffman and Forcada 2012). As a result, it is possible or even likely that chemical communication will influence the local relatedness structure of fur seal breeding colonies with downstream impacts on inbreeding and mate choice (Hoffman et al. 2007; Humble et al. 2020). On the other hand, chemical similarities between mothers and their pups are consistent with the hypothesis that mother-offspring recognition in this species may involve self-referent phenotype matching, a conceptually simple mechanism whereby an individual's own phenotype is used as a template for the recognition of close relatives (Blaustein, 1983). 
177 years later, collecting and analysing chemical samples from 50 new mother-offspring pairs using 178 virtually identical methodology. Because these two studies were carried out five years apart, none 179 of the individuals overlapped, precluding analysis of the reproducibility of chemical patterns 180 within individuals. Instead, we use the term "reproducibility" to refer to the extent to which broad 181 chemical patterns, i.e. differences between colonies and similarities between mothers and their 182 offspring, can be replicated with non-overlapping samples from different time points.

183

184 In addition, we wanted to know whether chemical differences between animals from SSB and 185 FWB are specific to this particular experimental setting, or whether chemical signatures are 186 colony-specific in general. We therefore analysed chemical samples from an additional 60 pups 187 from four other colonies around Bird Island in order to test for the generality of the colony 188 membership pattern, by which we mean the extent to which chemical differences are more 189 generally present among animals from different colonies. We hypothesised that (i) the originally 190 reported patterns of colony membership and mother-offspring similarity would be repeatable; and 191 (ii) that animals from different breeding colonies would differ chemically from one another in 192 general. 


\section{Materials \& Methods}

195

196 Study site and fieldwork

197 Chemical samples were taken from six Antarctic fur seal breeding colonies on Bird Island, South 198 Georgia $\left(54^{\circ} 00^{\prime} \mathrm{S}, 38^{\circ} 02^{\prime} \mathrm{W}\right.$ ) during the peak of the 2016 breeding season (November to 199 December; the previous study was conducted during the peak of the 2011 breeding season). A 200

201

202 203

204

205

206

207

208

209

210

211

212

213 GC-MS profiling and data alignment

214 We first took $2 \mathrm{~mL}$ of each sample and allowed the ethanol to evaporate at room temperature for 215 a maximum of 12 hours before resuspending in $2 \mathrm{~mL}$ dichloromethane (DCM). After a further 216 total of 50 mother-offspring pairs (including one pair of twins) were sampled from SSB and FWB as part of annual routine procedures of the long-term monitoring and survey program of the British Antarctic Survey (BAS). Additional samples were collected from a total of 60 pups from four colonies (15 samples each from Johnson Cove, Main Bay, Landing Beach and Natural Arch, Figure 1). Here, pups were opportunistically sampled from areas of the beach that were easily accessible. Adult females and pups were captured and restrained on land using standard methodology (Gentry \& Holt, 1982). Chemical samples were obtained by rubbing the cheek underneath the eye and behind the snout with sterile cotton wool swabs, which were stored individually at $-20^{\circ} \mathrm{C}$ in glass vials containing approximately $10 \mathrm{~mL}$ of $60 \% / 40 \%$ (vol/vol) ethanol/water. All of the chemical samples were collected immediately after capture by the same team of experienced field scientists. The samples were frozen at the latest one hour after collection and were stored for approximately 18 months prior to analysis. evaporation step, in which the DCM was reduced to a final volume of approximately $100 \mu \mathrm{L}$, the samples were analysed on a GC with a VF5-MS column (30 m x $0.25 \mathrm{~mm}$ inner diameter, $10 \mathrm{~m}$ guard column; Agilent Technologies, Santa Clara, USA) connected to a mass spectrometer (GCMS-QP2020, Shimadzu, Kyoto, Japan). One $\mu \mathrm{L}$ of each sample was injected into a deactivated glass-wool-packed liner with an inlet temperature of $225^{\circ} \mathrm{C}$. A split ratio of 3.2 was used and the carrier gas (Helium) flow rate was held constant at $1.2 \mathrm{~mL} / \mathrm{min}$. The GC run started with three minutes at $60{ }^{\circ} \mathrm{C}$ and then ramped up in increments of $10{ }^{\circ} \mathrm{C} / \mathrm{min}$ to reach a final temperature of $280^{\circ} \mathrm{C}$, which was maintained for 30 minutes. Mass spectra were taken in electron ionization mode with five scans per second in full scan mode $(50-600 \mathrm{~m} / \mathrm{z})$. The resulting GC-MS 
225 data were then processed using OpenChrom (Wenig \& Odermatt, 2010) for detection and 226 correction of split peaks. Afterwards, we used GCalignR in R (Ottensmann et al., 2018; R Core 227 Team, 2019) to align the resulting chromatograms by correcting minor shifts in retention times 228 among samples and maximizing the number of shared components.

229

230 Data visualisation and statistical analysis

231 Prior to data analyses, we excluded any compounds that were only observed in a single seal sample. 232 We then used non-metric multidimensional scaling (NMDS) to visualize the chemical data. This 233 approach reduces dimensionality so that each individual data point can be placed in a 2D 234 scatterplot where ranked between-individual distances are preserved and individuals that are 235 chemically more similar are closer together. NMDS was performed on a $\log (\mathrm{x}+1)$ transformed 236 relative abundance matrix comprising pairwise Bray-Curtis similarity values. We tested for 237 differences among and between a priori defined groups (i.e. the breeding colonies and mother238 offspring pairs) using a non-parametric permutational multivariate analysis of variance 239 (PERMANOVA). PERMANOVA tests whether the centroids of pre-defined groups differ 240 statistically for a chosen distance measure. It compares within-group to among-group variance 241 components and assigns statistical significance based on random permutations of objects within 242 groups. Each PERMANOVA was based on 99,999 permutations, although comparable results 243 were also obtained with 9999, 999 and 99 permutations. To determine whether differences 244 between our pre-defined groups were purely attributable to compositional differences between 245 groups rather than compositional differences within groups, we used the 'betadisper' function in 246 the vegan package in $\mathrm{R}$ to analyse the multivariate homogeneity of group dispersions (Oksanen et 247 al., 2019). In addition, we performed pairwise PERMANOVAs for different groups within the 248 model strata based on age and colony and Bonferroni corrected the resulting $p$-values.

249

250 Quantification of the explained variance

251 To facilitate a comparison of our effect sizes with those reported by Stoffel et al. (2015), we 252 quantified the proportion of the total chemical variance attributable to colony membership and 253 family ID in both studies. The scripts that Stoffel et al. (2015) used to align their data have now 254 been embedded into GCalignR and are therefore consistent between the two studies. As different 255 chemical datasets will have different optimal parameter settings for the alignment algorithm, we 
256 did not re-align or adjust the dataframe of Stoffel et al. (2015). Enforcing the same parameter 257 settings as in the current study would almost certainly lead to a loss of data quality and result in 258 artificially reduced effect sizes. To standardise effect size estimates between the studies, both 259 chemical datasets were bootstrapped over individuals to generate 5,000 datasets per study, each 260 comprising 15 mother-offspring pairs from SSB and 15 pairs from FWB (i.e. a total of 60 261 individuals). PERMANOVA was then implemented separately for each dataset and the resulting $262 R^{2}$ values were extracted for each of the predefined groups.

263

264 Data availability

265 The raw chemical data generated during this study are available via Github and the data of Stoffel 266 et al. (2015) can be downloaded from https://github.com/mastoffel/seal_chemical_fingerprints. 267 All of the code used to analyse the raw data are available as a PDF file written in Rmarkdown (see 268 supplementary information). The full documented data analysis pipeline can be downloaded from 269 our GitHub repository at https://github.com/tebbej/SealScent2020/.

270

271 Ethical statement

272 Samples were collected as part of the Polar Science for Planet Earth program of the British 273 Antarctic Survey under the authorization of the Senior Executive and the Environment Officers of 274 the Government of South Georgia and the South Sandwich Islands (permit no. 2016/013). Samples 275 were collected and retained under Scientific Research Permits for the British Antarctic Survey 276 field activities on South Georgia, and in accordance with the Convention on International Trade in 277 Endangered Species of Wild Fauna and Flora. All field procedures were approved by the British 278 Antarctic Survey Animal Welfare and Ethics Review Body (reference no. PEA6). 279 


\section{Results}

281 In order to investigate the reproducibility of chemical patterns of colony membership and mother-

282 offspring similarity in Antarctic fur seals, we analysed chemical data from mother-offspring pairs 283 from SSB and FWB as well as pups from an additional four breeding colonies around Bird Island 284 (Figure 1). We detected an average of $42 \pm 15$ s.d. chemicals per sample. No significant 285 differences were found in the number of chemicals between mothers and offspring (unpaired t286 test, $t=0.8403, p=0.403$ ) or among pups from the six breeding colonies (ANOVA, $F_{5,104}=0.001$, $287 p=0.98)$.

288

289 Reproducibility of chemical patterns

290 Multivariate statistical analysis of the relative proportions of each substance revealed highly 291 significant differences between animals from SSB and FWB (PERMANOVA, $p<0.0001$, Figure 292 2a, Table 1a). A highly significant effect of mother-pup pair ID nested within colony 293 (PERMANOVA, $p<0.0001$, Figure 2b, Table 1a) was also found, indicating that mothers and 294 their pups are chemically more similar to one another than expected by chance. A test for 295 multivariate homogeneity of group variances uncovered marginally significant differences among 296 the groups ( $p=0.026$, Table 1a), which could potentially indicate the involvement of additional 297 explanatory factors that were not accounted for in the model. We therefore investigated further by 298 splitting the chemical data into four groups, corresponding to mothers and pups from SSB and 299 FWB respectively. Performing PERMANOVAs for all possible pairwise combinations of these 300 groups produced three important outcomes. First, all of the pairwise PERMANOVAs involving 301 groups of animals from the two different colonies were highly significant after table-wide 302 Bonferroni correction for multiple tests (Table S1). This indicates that colony membership is 303 chemically encoded irrespective of whether individuals are mothers or pups. Second, both of the pairwise PERMANOVAs involving mothers and pups within colonies were non-significant after 305 Bonferroni correction (Table S1). This suggests that mothers and their pups are chemically similar to one another, regardless of the colony in question. Finally, tests for the homogeneity of group variances were not significant for any of the pairwise group comparisons after Bonferroni 308 correction (Table S2). This implies that our results are unlikely to be driven by differences in chemical variance among groups. 
311 As $p$-values cannot be directly compared between studies with different sample sizes, we used the

312 PERMANOVA framework to estimate the effect sizes of colony membership and mother313 offspring similarity in both studies. To facilitate direct comparisons while also incorporating 314 uncertainty due to chemical variation among individuals, both datasets were bootstrapped over 315 individuals as described in the Materials and methods. We found that effect size estimates for 316 colony membership and mother-offspring similarity (maximum density $R^{2}$ values) differed by only 317 few percent between the two studies (Figure 3) and consistently fell within the range of $0.08<R^{2}$ $318<0.15$.

319

320 Generality of chemical patterns

321 To investigate whether chemical signatures are colony-specific in general, we analysed chemical 322 data from pups sampled from a total of six colonies around Bird Island. PERMANOVA uncovered 323 chemical differences not only between SSB and FWB, but also more generally among colonies 324 (Figure S1). These differences were statistically significant both overall $(p<0.0001$, Table 1b) 325 and for the majority of pairwise comparisons after Bonferroni correction (Table 2). 


\section{Discussion}

328 A major obstacle to reproducible research in ecology and evolution is the perceived difficulty of

329 replicating original research findings in natural settings where many variables cannot be controlled 330 for and where spatial and temporal dependencies may confound faithful replication attempts 331 (Nakagawa \& Parker, 2015; Fidler et al., 2017). Although the inherent variability of natural 332 systems undoubtedly poses a challenge to replication studies, our findings suggest that, at least 333 under some circumstances, chemical patterns may be repeatable. Specifically, we found that the 334 effect sizes of patterns of colony membership and mother-offspring similarity in Stoffel et al. 335 (2015) were of similar magnitude in a new sample of mother-offspring pairs separated by five 336 years. By expanding the geographical scope of our sampling, we could furthermore show that 337 chemical signatures are colony-specific in general. Our results lend further support to the 338 conclusion that colony membership and mother-offspring similarity are chemically encoded in 339 Antarctic fur seals.

340

\section{Motivation and study design}

342 A number of factors motivated the current replication attempt. First, the results of Stoffel et al. 343 (2015) were based on a modest sample of Antarctic fur seal mother-offspring pairs sampled in a 344 single season. We therefore wanted to safeguard against type I error while also testing for the 345 repeatability of chemical patterns over time. Second, chance results can become highly influential 346 (Kelly, 2006) and our original study already appears to have motivated comparable investigations 347 in other pinniped species. For example, a recent study of Australian sea lions using a very similar 348 experimental design also reported chemical differences between two breeding colonies, but 349 chemical similarities were not found between mothers and their pups (Wierucka et al., 2019). 350 Although it is not unreasonable to assume that different species might vary in how chemical 351 information is encoded and used in mother-offspring recognition, this point of difference 352 nevertheless encouraged us to revisit our original findings. Finally, being able to confirm and 353 extend our original results strengthens the case for follow-up studies and reduces the risk of time 354 and resources being wasted on chasing up false positives.

Although we acknowledge that no study of a wild population can ever be perfectly replicated 357 (Nakagawa \& Parker, 2015; Fidler et al., 2017), we believe that our replication study of chemical 
358 patterns in Antarctic fur seals is sufficiently close to that of Stoffel et al. (2015) in terms of both 359 experimental design and implementation to be considered an exact replication. In practice, there 360 were a handful of small differences between the two studies, but these were mainly a consequence 361 of incremental improvements to our methodology and are unlikely to have had a major influence 362 on the final outcome. For example, because replication studies often produce smaller effect sizes 363 than original studies (Simonsohn, 2015; Open science collaboration, 2015), we attempted to 364 enlarge our sample size of mother-offspring pairs as far as was practicable. We also improved the 365 standardization and reproducibility of our chemical analysis pipeline by performing peak detection 366 with open source software and by integrating the alignment algorithm of Stoffel et al. (2015) into 367 an R package (Ottensmann et al., 2018). However, these small modifications appear to have been

368 369 370

371 372 373

374 375 376 377 378 379 380

381 382 383 384 385

386 387 of little consequence as the effect sizes of colony membership and mother-offspring similarity did not differ systematically between the two studies.

Two further methodological differences were beyond our control. First, owing to the fact that the original and replication studies were carried out five years apart, the sampling was conducted by different teams of field biologists. However, we used carefully standardized field protocols in order to minimize any inadvertent experimental variation. Second, the GC-MS machine used by Stoffel et al. (2015) was subsequently replaced by a newer and more sensitive model. One might have expected this to result in more chemicals being detected in the replication study, which would be expected to provide greater power to detect chemical patterns. If anything, however, fewer chemicals in total were detected in the current study, possibly because of differences in the concentrations of samples or because we used different peak calling software and manually curated the resulting dataset to remove redundant split peaks. Regardless of the exact explanation, the overall similarity of the results of the two studies suggests that patterns of colony membership and mother-offspring similarity in Antarctic fur seals are robust to these minor sources of experimental variation. This robustness would be expected if chemical patterns are influenced by large numbers of compounds and therefore persist independently of minor methodological differences that may influence which subsets of peaks are detected and retained for analysis.

Replication outcomes 
388 Successful replication can be defined either in the context of statistical significance (Rosenthal, 389 1991) or on the basis of a comparison of effect sizes (Goodman, Fanelli \& Ioannidis, 2016; Piper 390 et al., 2019). We not only tested for significance but also developed an approach based on 391 PERMANOVA to evaluate the effect sizes of colony membership and mother-offspring similarity 392 in both datasets. Specifically, we extracted $R^{2}$ values for the terms in question after bootstrapping 393 both chemical datasets over individuals. This approach controlled for differences in sample size 394 between the two studies while also providing a visual representation of the magnitude of 395 uncertainty associated with the $R^{2}$ estimates. We not only found that the patterns reported by 396 Stoffel et al. (2015) remained highly significant, but also that the effect size estimates of colony 397 membership and mother-offspring similarity in the two studies were more or less similar, varying 398 by at most a few percent. Elsewhere, in a study that attempted to replicate a hundred psychological 399

400

401

402

403

404

405 Generality of the colony membership pattern

406 We went a step beyond simply repeating our previous study by investigating whether chemical 407 408 409 410 411

412

413

414

415 416 417 418 as $p$-values, was more predictive of replication success than other characteristics such as the experience or expertise of the original and replication teams. This is consistent with the outcome of the current replication exercise given the high significance $(p<0.0001)$ of the patterns originally reported by Stoffel et al. (2015).

differences between SSB and FWB are specific to these two colonies, or whether chemical signatures are colony-specific in general. Unfortunately, it was not possible to sample mothers from locations other than SSB and FWB due to the difficulty of capturing adult females farther away from the BAS field station where fieldwork on seals is rarely if ever performed. However, the relative ease of capturing pups enabled us to gather a more representative collection of chemical samples from multiple breeding sites around Bird Island. After controlling for the false discovery rate, statistically significant chemical differences were detected in all but two out of 15 pairwise comparisons between colonies. This suggests not only that chemical patterns of colony membership are repeatable over time, but also that they can be generalized over space. Interestingly, we did not find a clear correspondence between chemical similarity and the geographical proximity of colonies. For example, Freshwater Beach and Main Bay were among the most chemically dissimilar colonies despite being only around 500m apart, while Johnson studies (Open science collaboration, 2015), variation in the strength of the original evidence, such 
419 Cove and Natural Arch were among the most chemically similar colonies despite being situated at

420 the opposite extremes of Bird Island. The most probable explanation for this pattern is that 421 chemical differences among colonies are predominantly shaped by as yet unknown environmental

422 factors (see below).

423

424 Mechanisms encoding chemical information

425 Relatively little is currently known about the mechanisms by which colony membership and 426 mother-offspring similarity are chemically encoded in Antarctic fur seals. We know that animals 427 from SSB and FWB exhibit chemical differences despite a lack of genetic differentiation (Stoffel 428 et al 2015), which implies that environmental drivers play an important role. However, it remains 429 unclear exactly what these drivers might be. Food is unlikely to be an important determinant of 430 colony-specific chemical patterns because breeding female fur seals feed predominantly on 431 Antarctic krill (Boyd, Staniland \& Martin, 2002). The underlying substrate is also relatively 432 homogenous, with the vast majority of animals occupying cobblestone breeding beaches that show 433 little in the way of obvious differences to the human eye. It is therefore more likely that colony434 specific chemical phenotypes are influenced by differences in local conditions such as temperature, 435 wind or solar radiation, either directly or via alterations to the skin microbiota (Grosser et al 2019). 436 A further possibility could be that chemical differences between colonies reflect differences in 437 microbial communities shaped by social stress. For example, stressful conditions such as high 438 densities of conspecifics can suppress microbial diversity (Bailey et al., 2011; Stothart et al., 2016; 439 Noguera et al., 2018; Partrick et al., 2018; Zha et al., 2018). This is consistent with our data, as 440 breeding females on SSB are present at higher density and have chronically elevated levels of the 441 stress hormone cortisol (Meise et al., 2016), while skin microbial diversity is also lower in this 442 colony (Grosser et al 2019). Investigating the potential linkages between social stress, cortisol, 443 microbial community structure and chemical phenotypes represents a promising avenue for future 444 research.

\section{Conclusions}

447 Our study set out to test two hypotheses, namely that chemical patterns of colony membership and 448 mother-offspring similarity in Antarctic fur seals are reproducible over time, and that chemical 449 differences will be present not only between SSB and FWB, but also more generally among 
450 colonies. Both hypotheses were supported by our data. The overall robustness of chemical 451 patterns of colony membership and mother-offspring similarity in Antarctic fur seals is consistent 452 with the notion that chemical information could be important for social communication in 453 pinnipeds, and lays a solid foundation for future studies of the mechanisms responsible for 454 chemical variation. Finally, as a lack of access to raw data, code and software has been identified 455 as a fundamental obstacle to replication (Fidler et al., 2017), we have made the data from both 456 studies as well as the code used to analyze them freely available, while also using maximally 457 transparent, open access software for peak detection and alignment.

458

\section{Acknowledgements}

460 We are grateful to the field assistants on Bird Island who contributed toward animal handling and 461 tissue sampling. We would also like to thank John Dickens who helped collect samples from 462 additional beaches on Bird Island. This work contributes to the Ecosystems project of the British 463 Antarctic Survey, Natural Environmental Research Council, and is part of the Polar Science for 464 Planet Earth Programme. 


\section{References}

467 Bailey MT, Dowd SE, Galley JD, Hufnagle AR, Allen RG, Lyte M. 2011. Exposure to a social

468

469

470

471

472

473

474

475

476

477

478

479

480

481

482

483

484

485

486

487

488

489

490

491

492

493

494

495

496

stressor alters the structure of the intestinal microbiota: Implications for stressor-induced immunomodulation. Brain, Behavior, and Immunity 25:397-407. DOI:

10.1016/j.bbi.2010.10.023.

Baker M. 2016. 1,500 scientists lift the lid on reproducibility. Nature 533:452-454. DOI: $10.1038 / 533452 \mathrm{a}$.

Begley CG, Ellis LM. 2012. Raise standards for preclinical cancer research. Nature 483:531533. DOI: $10.1038 / 483531 \mathrm{a}$.

Blaustein AR. 1983. Kin recognition mechanisms: Phenotypic matching or recognition alleles? The American Naturalist 121:749-754. DOI: 10.1086/284101.

Boyd I, Staniland I, Martin A. 2002. Distribution of foraging by female Antarctic fur seals. Marine Ecology Progress Series 242:285-294. DOI: 10.3354/meps242285.

Burgener N, Dehnhard M, Hofer H, East ML. 2009. Does anal gland scent signal identity in the spotted hyaena? Animal Behaviour 77:707-715. DOI: 10.1016/j.anbehav.2008.11.022.

Caspers BA, Schroeder FC, Franke S, Streich WJ, Voigt CC. 2009. Odour-based species recognition in two sympatric species of sac-winged bats (Saccopteryx bilineata, S. leptura): combining chemical analyses, behavioural observations and odour preference tests. Behavioral Ecology and Sociobiology 63:741-749. DOI: 10.1007/s00265-009-0708-7.

Caspers BA, Schroeder FC, Franke S, Voigt CC. 2011. Scents of adolescence: the maturation of the olfactory phenotype in a free-ranging mammal. PLOS ONE 6:e21162. DOI: 10.1371/journal.pone.0021162.

Charpentier MJE, Boulet M, Drea CM. 2008. Smelling right: the scent of male lemurs advertises genetic quality and relatedness. Molecular Ecology 17:3225-3233.

Dirnagl U. 2019. Rethinking research reproducibility. The EMBO Journal 38:2018-2020. DOI: 10.15252/embj.2018101117.

Dobson FS, Jouventin P. 2003. How mothers find their pups in a colony of Antarctic fur seals. Behavioural Processes 61:77-85. DOI: 10.1016/S0376-6357(02)00164-X.

Fidler F, En Chee Y, Wintle BC, Burgman MA, McCarthy MA, Gordon A. 2017. Metaresearch for evaluating reproducibility in ecology and evolution. BioScience 67:282-289. DOI: 10.1093/biosci/biw159.

Peer) reviewing PDF | (2020:04:48370:1:3:NEW 10 Aug 2020) 
497 Fisher RA. 1974. The design of experiments. New York: Hafner Press.

498 Fraser H, Parker T, Nakagawa S, Barnett A, Fidler F. 2018. Questionable research practices in 499 ecology and evolution. PLOS ONE 13:e200303. DOI: 10.1371/journal.pone.0200303.

500 Fratini G, Lois S, Pazos M, Parisi G, Medina I. 2012. Volatile profile of Atlantic shellfish

501

502

503

504

505

506

507

508

509

510

511

512

513

514

515

516

517

518

519

520

521

522

523

524

525

526

527 species by HS-SPME GC/MS. Food Research International 48:856-865. DOI: 10.1016/j.foodres.2012.06.033.

Goodman SN, Fanelli D, Ioannidis JPA. 2016. What does research reproducibility mean? Science translational medicine 8. DOI: 10.1126/scitranslmed.aaf5027

Grosser S, Sauer J, Paijmans AJ, Caspers BA, Forcada J, Wolf JBW, Hoffman JI. 2019. Fur seal microbiota are shaped by the social and physical environment, show mother-offspring similarities and are associated with host genetic quality. Molecular Ecology 28:2406-2422. DOI: $10.1111 / \mathrm{mec} .15070$.

Hamilton JE. 1956. Scent of otariids. Nature 177:900.

Hardy MH, Roff E, Smith TG, Ryg M. 1991. Facial skin glands of ringed and grey seals, and their possible function as odoriferous organs. Canadian Journal of Zoology 69:189-200. DOI: 10.1139/z91-029.

Hoffman JI, Trathan PN, Amos W. 2006. Genetic tagging reveals extreme site fidelity in territorial male Antarctic fur seas Arctocephalus gazella. Molecular Ecology 15: 3841-3847. DOI: $10.1111 / \mathrm{j} .1365-294 X .2006 .03053 . \mathrm{x}$

Hoffman JI, Forcada J, Trathan PN, Amos W. 2007. Female fur seals show active mate choice for males that are heterozygous and unrelated. Nature 445: 912-914. DOI: 10.1038 /nature 05558

Hoffman JI, Forcada J. 2012. Extreme natal philopatry in female Antarctic fur seals (Arctocephalus gazella). Mammalian Biology 77: 71-73. DOI: 10.1016/j.mambio.2011.09.002

Hurst JL, Beynon RJ. 2010. Making progress in genetic kin recognition among vertebrates. Journal of biology 9:13. DOI: 10.1186/jbiol221

Jennions MD, Møller AP. 2002. Publication bias in ecology and evolution: an empirical assessment using the 'trim and fill'method. Biological Reviews 77:211-222. DOI: https://doi.org/10.1017/S1464793101005875

Kean EF, Chadwick EA, Mueller CT. 2015. Scent signals individual identity and country of 
528

529

530

531

532

533

534

535

536

537

538

539

540

541

542

543

544

545

546

547

548

549

550

551

552

553

554

555

556

557

558

origin in otters. Mammalian Biology 80:99-105. DOI: 10.1016/j.mambio.2014.12.004.

Kean EF, Müller CT, Chadwick EA. 2011. Otter scent signals age, sex, and reproductive status. Chemical Senses 36:555-564. DOI: 10.1093/chemse/bjr025.

Kelly CD. 2006. Replicating empirical research in behavioral ecology: how and why it should be done but rarely ever is. The Quarterly review of biology 81:221-236. DOI: https://doi.org/10.1086/506236

Kelly CD. 2019. Rate and success of study replication in ecology and evolution. PeerJ 7:e7654. DOI: $10.7717 /$ peerj.7654.

Kishida T, Kubota S, Shirayama Y, Fukami H. 2007. The olfactory receptor gene repertoires in secondary-adapted marine vertebrates: evidence for reduction of the functional proportions in cetaceans. Biology Letters 3:428-430. DOI: 10.1098/rsbl.2007.0191.

Kohlwey S, Krause ET, Baier MC, Müller C, Caspers BA. 2016. Chemical analyses reveal family-specific nest odor profiles in zebra finches (Taeniopygia guttata): A pilot study. In: Chemical Signals in Vertebrates 13. Cham: Springer International Publishing, 167-175. DOI: 10.1007/978-3-319-22026-0_12.

Kovacs KM. 1995. Mother-pup reunions in harp seals, Phoca groenlandica - cues for the relocation of pups. Canadian Journal of Zoology -Revue Canadienne de Zoologie 73:843849. DOI: $10.1139 / \mathrm{z} 95-099$.

Kowalewsky S, Dambach M, Mauck B, Dehnhardt G. 2006. High olfactory sensitivity for dimethyl sulphide in harbour seals. Biology Letters 2:106-109. DOI: 10.1098/rsbl.2005.0380.

Krause ET, Brummel C, Kohlwey S, Baier MC, Müller C, Bonadonna F, Caspers BA. 2014. Differences in olfactory species recognition in the females of two Australian songbird species. Behavioral Ecology and Sociobiology 68:1819-1827. DOI: 10.1007/s00265-014$1791-y$.

Ling J, K. 1974. The integument of marine mammals. Functional Anatomy of Marine Mammals $2: 1-44$.

Meise K, von Engelhardt N, Forcada J, Hoffman JI. 2016. Offspring hormones reflect the maternal prenatal social environment: Potential for foetal programming? PLOS ONE 11:e0145352. DOI: 10.1371/journal.pone.0145352.

Mendoza D, Garcia CA. 2017. Defining research reproducibility: what do you mean? Clinical 
559

560

561

562

563

564

565

566

567

568

569

570

571

572

573

574

575

576

577

578

579

580

581

582

583

584

585

586

587

588

589

Chemistry 63:1777. DOI: https://doi.org/10.1373/clinchem.2017.279984

Müller T, Müller C. 2016. Consequences of mating with siblings and nonsiblings on the reproductive success in a leaf beetle. Ecology and Evolution 6:3185-3197. DOI: 10.1002/ece3.2103.

Nakagawa S, Parker TH. 2015. Replicating research in ecology and evolution: feasibility, incentives, and the cost-benefit conundrum. BMC Biology 13:1-6. DOI: 10.1186/s12915015-0196-3.

Noguera JC, Aira M, Pérez-Losada M, Domínguez J, Velando A. 2018. Glucocorticoids modulate gastrointestinal microbiome in a wild bird. Royal Society Open Science 5:171743. DOI: $10.1098 /$ rsos. 171743.

Oksanen J, Blanchet FG, Friendly M, Kindt R, Legendre P, McGlinn D, Minchin PR, O'Hara RB, Simpson GL, Solymos P, Stevens MHH, Szoecs E, Wagner H. 2019. vegan: Community Ecology Package.

Open science collaboration. 2015. Estimating the reproducibility of psychological science. Science 349:aac4716-aac4716. DOI: 10.1126/science.aac4716.

Ottensmann M, Stoffel MA, Nichols HJ, Hoffman JI. 2018. GCalignR: An R package for aligning gas-chromatography data for ecological and evolutionary studies. PLOS ONE 13:120. DOI: 10.1371/journal.pone.0198311.

Palmer AR. 2000. Quasi-replication and the contract of error: lessons from sex ratios, heritabilities and fluctuating asymmetry. Annual Review of Ecology and Systematics 31:441-480. DOI: 10.1146/annurev.ecolsys.31.1.441.

Partrick KA, Chassaing B, Beach LQ, McCann KE, Gewirtz AT, Huhman KL. 2018. Acute and repeated exposure to social stress reduces gut microbiota diversity in Syrian hamsters. Behavioural Brain Research 345:39-48. DOI: 10.1016/j.bbr.2018.02.005.

Phillips A V. 2003. Behavioral cues used in reunions between mother and pup south american fur seals (Arctocephalus australis). Journal of Mammalogy 84:524-535. DOI: 10.1644/1545-1542(2003)084<0524:BCUIRB >2.0.CO;2.

Piper SK, Grittner U, Rex A, Riedel N, Fischer F, Nadon R, Siegerink B, Dirnagl U. 2019. Exact replication: foundation of science or game of chance? PLoS Biology 17:1-9. DOI: 10.1371/journal.pbio.3000188.

Pitcher BJ, Harcourt RG, Schaal B, Charrier I. 2011. Social olfaction in marine mammals: wild 
590

591

592

593

594

595

596

597

598

599

600

601

602

603

604

605

606

607

608

609

610

611

612

613

614

615

616

617

618

619

620

female Australian sea lions can identify their pup's scent. Biology Letters 7:60-62. DOI: 10.1098/rsbl.2010.0569.

R Core Team. 2019. R: A language and environment for statistical computing.

Rosenthal R. 1991. Replication in behavioural research. In: Neuliep JW ed. Replication research in the social sciences. Newbury Park [u.a.]: Sage Publ., 1-31.

Schmidt S. 2009. Shall we really do it again? The powerful concept of replication is neglected in the social sciences. Review of general psychology 13:90-100. DOI:

https://doi.org/10.1037/a0015108

Schneeberger K, Voigt CC, Müller C, Caspers BA. 2016. Multidimensionality of chemical information in male greater sac-winged bats (Saccopteryx bilineata). Frontiers in Ecology and Evolution 4. DOI: 10.3389/fevo.2016.00083.

Setchell JM, Vaglio S, Abbott KM, Moggi-Cecchi J, Boscaro F, Pieraccini G, Knapp LA. 2011. Odour signals major histocompatibility complex genotype in an Old World monkey. Proceedings of the Royal Society B: Biological Sciences 278:274-280. DOI: 10.1098/rspb.2010.0571.

Simonsohn U. 2015. Small telescopes. Psychological Science 26:559-569. DOI: $10.1177 / 0956797614567341$.

Stoffel MA, Caspers BA, Forcada J, Giannakara A, Baier M, Eberhart-Phillips L, Müller C, Hoffman JI. 2015. Chemical fingerprints encode mother-offspring similarity, colony membership, relatedness, and genetic quality in fur seals. Proceedings of the National Academy of Sciences 112:E5005-E5012. DOI: 10.1073/pnas.1506076112.

Stothart MR, Bobbie CB, Schulte-Hostedde AI, Boonstra R, Palme R, Mykytczuk NCS, Newman AEM. 2016. Stress and the microbiome: linking glucocorticoids to bacterial community dynamics in wild red squirrels. Biology Letters 12:20150875. DOI: 10.1098/rsbl.2015.0875.

Sun L, Müller-Schwarze D. 1998. Anal gland secretion codes for family membership in the beaver. Behavioral Ecology and Sociobiology 44:199-208.

Vogt K, Boos S, Breitenmoser U, Kolliker M. 2016. Chemical composition of Eurasian lynx urine conveys information on reproductive state, individual identity, and urine age. Chemoecology 26:205-217. DOI: 10.1007/s00049-016-0220-2.

Wierucka K, Barthes N, Harcourt R, Schaal B, Charrier I, Pitcher BJ. 2019. Chemical 
621 fingerprints suggest direct familiarisation rather than phenotype matching during olfactory 622 recognition in Australian sea lions (Neophoca cinerea). Journal of Experimental Marine 623 Biology and Ecology 517:49-53. DOI: 10.1016/j.jembe.2019.06.001.

624 Wyatt TD. 2015. The search for human pheromones: the lost decades and the necessity of 625 returning to first principles. Proceedings of the Royal Society B: Biological Sciences 626 282:20142994. DOI: 10.1098/rspb.2014.2994.

627 Yamazaki K, Beauchamp GK, Imai Y, Bard J, Phelan SP, Thomas L, Boyse EA. 1990.

628 Odortypes determined by the major histocompatibility complex in germfree mice.

629 Proceedings of the National Academy of Sciences of the United States of America 87:8413630 8416. DOI: 10.1073/pnas.87.21.8413.

631 Zha Y, Eiler A, Johansson F, Svanbäck R. 2018. Effects of predation stress and food ration on 632 perch gut microbiota. Microbiome 6:28. DOI: 10.1186/s40168-018-0400-0. 


\section{Figure 1}

Locations of six Antarctic fur seal breeding colonies on Bird Island, South Georgia, where chemical samples were taken.

Mother-offspring pairs were sampled from the special study beach (SSB) and freshwater beach (FWB), whereas only pups were sampled from the other four colonies.

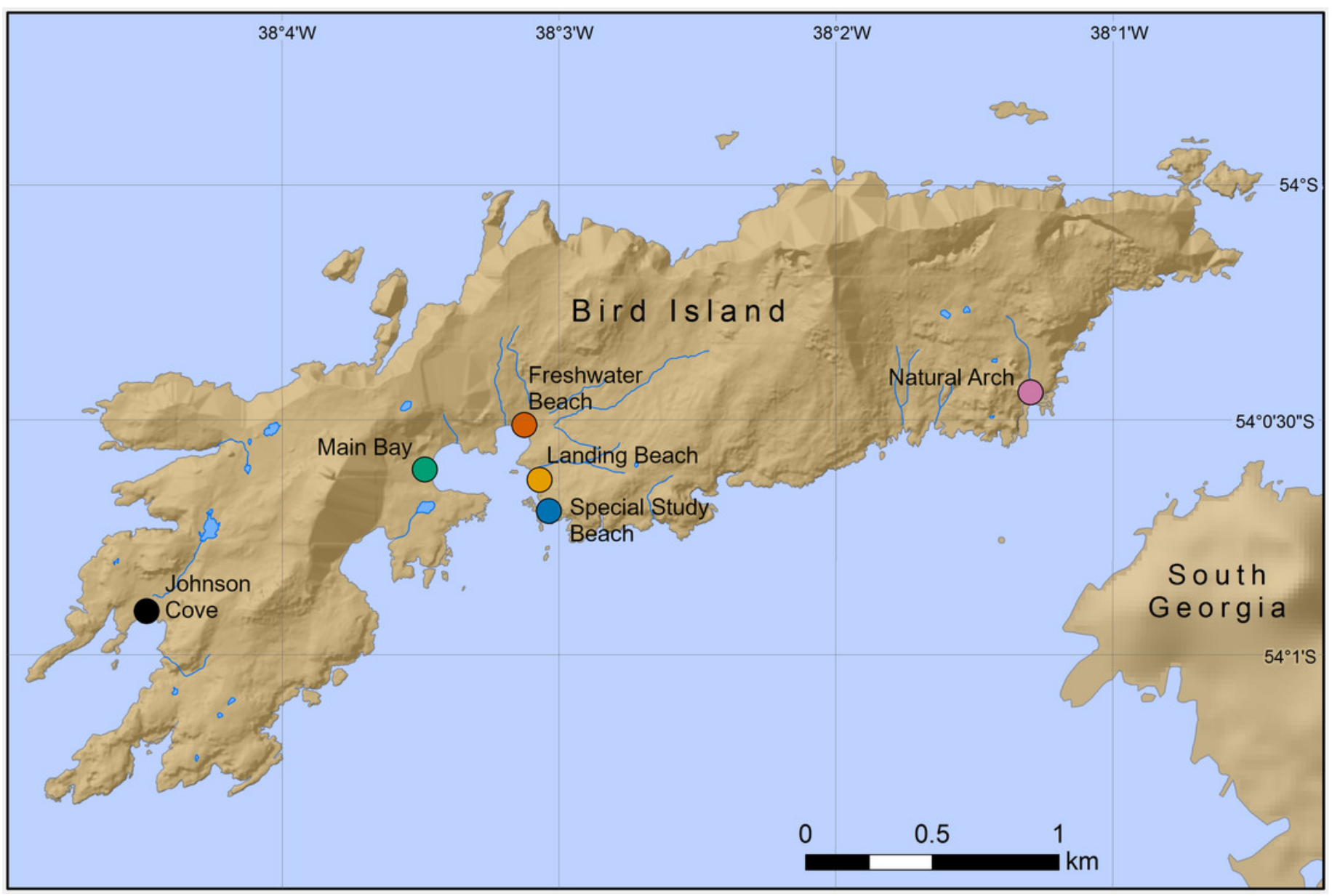




\section{Figure 2}

Two-dimensional non-metric multidimensional scaling (NMDS) plots of chemical data from skin swabs of Antarctic fur seal mother-offspring pairs from SSB and FWB.

NDMS was performed using Bray-Curtis similarity values calculated from $\log (x+1)$

transformed chemical abundance data. The scales of the two axes are arbitrary and the closer two points appear in the plot, the more similar they are chemically. Individual data points in panel $\mathbf{A}$ are colour-coded by colony (SSB $=$ blue, FWB $=$ red) and age (mother $=$ filled, offspring $=$ empty). Panel $\mathbf{B}$ shows mother offspring pairs, which are depicted by unique combinations of symbols and colours. The three blue triangles correspond to a mother on SSB with twin pups. 

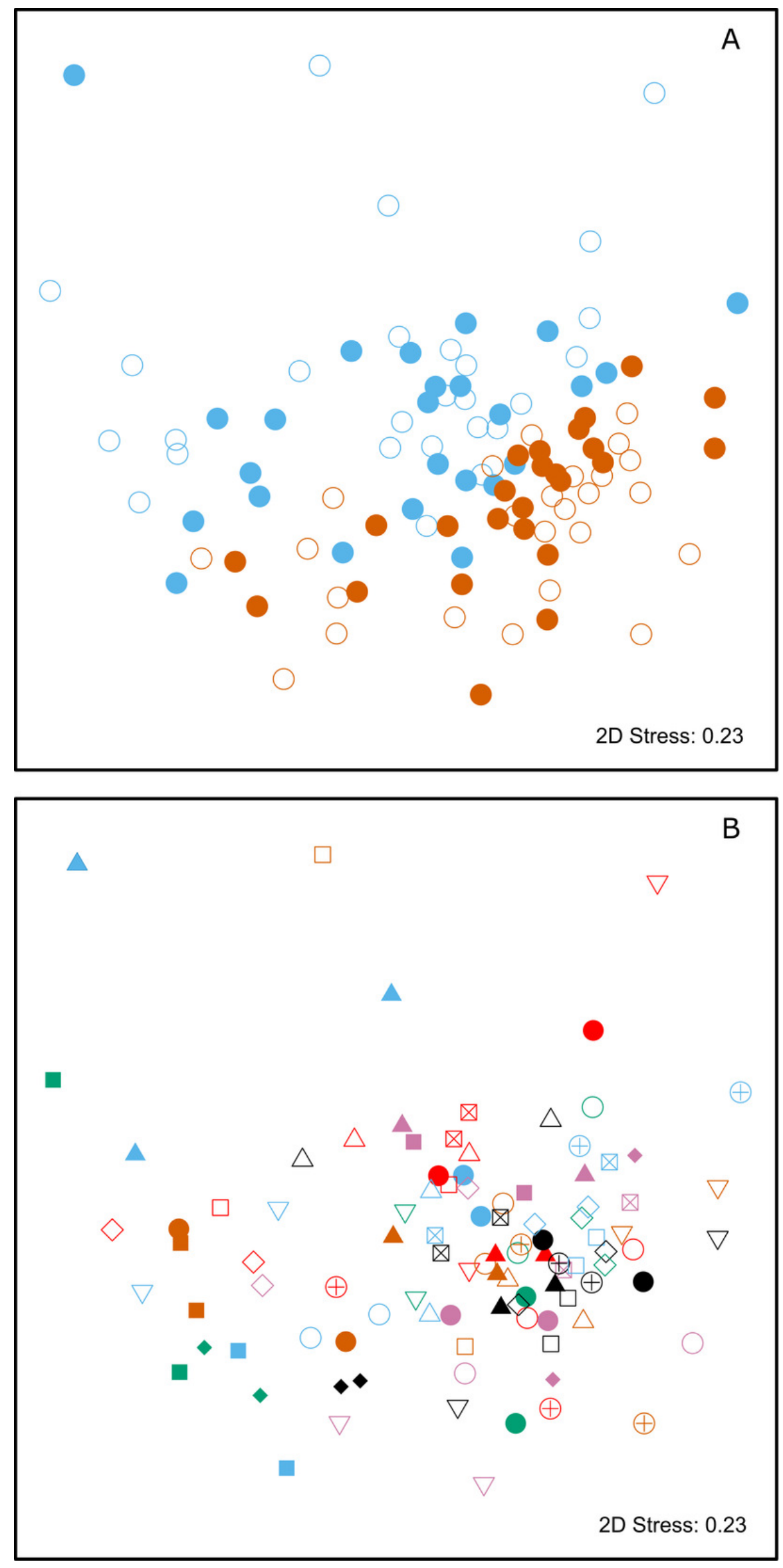

Peer) reviewing PDF | (2020:04:48370:1:3:NEW 10 Aug 2020) 


\section{Table $\mathbf{1}$ (on next page)}

Results of PERMANOVAs.

Results of PERMANOVAs of (a) 50 mother-offspring pairs from two colonies (SSB and FWB); and (b) 110 pups from six colonies. For comparison, a re-analysis of the chemical data of Stoffel et al. (2015) is shown in part (c). See Materials and methods for details. 


\begin{tabular}{|c|c|c|c|}
\hline $\begin{array}{l}\text { (a) PERMANOVA of mothers and offspring from two } \\
\text { colonies }\end{array}$ & $F$ & $R^{2}$ & $p$-value \\
\hline Age & 2.65 & 0.023 & 0.004 \\
\hline Colony membership & 9.07 & 0.076 & $<0.0001$ \\
\hline Family ID nested within colony membership & 9.02 & 0.153 & $<0.0001$ \\
\hline Test for homogeneity of variance for colony membership & 5.14 & - & 0.026 \\
\hline Test for homogeneity of variance for age & 1.47 & & 0.228 \\
\hline $\begin{array}{l}\text { Test for homogeneity of variance for age \& colony } \\
\text { membership }\end{array}$ & 1.91 & & 0.134 \\
\hline (b) PERMANOVA of pups from six colonies & $F$ & $R^{2}$ & $p$-value \\
\hline Colony membership & 5.17 & 0.191 & $<0.0001$ \\
\hline Test for homogeneity of variance for colony membership & 0.50 & - & 0.778 \\
\hline $\begin{array}{l}\text { (c) PERMANOVA of mothers and offspring from two } \\
\text { colonies (data from Stoffel et al. 2015). }\end{array}$ & $F$ & $\boldsymbol{R}^{2}$ & $p$-value \\
\hline Age & 0.98 & 0.010 & 0.461 \\
\hline Colony membership & 12.35 & 0.128 & $<0.0001$ \\
\hline Family ID nested within colony membership & 3.13 & 0.065 & $<0.0001$ \\
\hline Test for homogeneity of variance for colony membership & 0.22 & & 0.639 \\
\hline Test for homogeneity of variance for age & 0.35 & & 0.557 \\
\hline $\begin{array}{l}\text { Test for homogeneity of variance for age \& colony } \\
\text { membership }\end{array}$ & 0.21 & & 0.887 \\
\hline
\end{tabular}




\section{Figure 3}

Effect sizes of colony membership and mother-offspring similarity in the original study (Stoffel et al. 2015) and in this replication study.

To quantify the amount of explained variance, we bootstrapped both datasets over individuals and extracted the corresponding $R^{2}$ values for each of the predefined groups in separate PERMANOVAs (see Materials and methods for details). The data are presented as sinaplots with overlaid boxplots (centre line $=$ median, bounds of box $=25$ th and 75th percentiles, upper and lower whiskers = largest and lowest value but no further than $1.5 *$ inter-quartile range from the hinge) and the grey points represent effect sizes based on the full datasets. 
Colony membership
original study

Colony membership replication study

Mother-offspring similarity original study

Mother-offspring similarity replication study

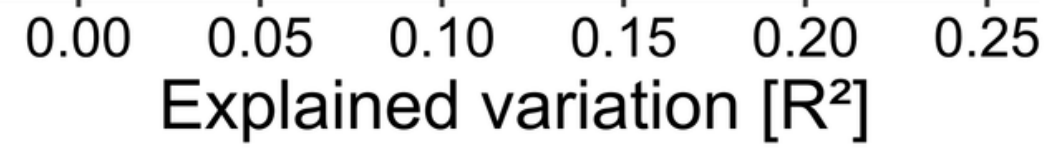




\section{Table 2 (on next page)}

Results of PERMANOVAs of colony membership in six pup colonies.

Results of PERMANOVAs of colony membership showing the magnitude and significance of chemical differences in all possible pairwise combinations of pups from six breeding colonies. See Materials and methods for details. 


\begin{tabular}{|l|c|c|c|c|}
\hline Pairs & $\boldsymbol{F}$ & $\boldsymbol{R}^{\mathbf{2}}$ & $\boldsymbol{p}$-value & Corrected $\boldsymbol{p}$-value \\
\hline SSB versus FWB & 6.08 & 0.110 & $<0.0001$ & $<0.001$ \\
SSB versus Landing Beach & 3.54 & 0.083 & $<0.001$ & 0.012 \\
SSB versus Main Bay & 6.18 & 0.137 & $<0.0001$ & $<0.0001$ \\
SSB versus Natural Arch & 4.17 & 0.097 & $<0.001$ & 0.002 \\
SSB versus Johnson Cove & 4.40 & 0.104 & $<0.0001$ & $<0.001$ \\
FWB versus Landing Beach & 4.16 & 0.099 & $<0.0001$ & $<0.001$ \\
FWB versus Main Bay & 7.29 & 0.161 & $<0.0001$ & $<0.001$ \\
FWB versus Natural Arch & 7.91 & 0.172 & $<0.0001$ & $<0.001$ \\
FWB versus Johnson Cove & 7.15 & 0.162 & $<0.0001$ & $<0.001$ \\
Landing Beach versus Main Bay & 3.32 & 0.106 & 0.002 & 0.030 \\
Landing Beach versus Natural Arch & 3.00 & 0.097 & 0.004 & 0.063 \\
Landing Beach versus Johnson Cove & 2.70 & 0.091 & 0.012 & 0.177 \\
Main Bay versus Natural Arch & 5.92 & 0.175 & $<0.0001$ & $<0.001$ \\
Main Bay versus Johnson Cove & 3.14 & 0.104 & $<0.001$ & 0.005 \\
Natural Arch versus Johnson Cove & 2.43 & 0.083 & 0.016 & 0.245 \\
\hline
\end{tabular}

